\title{
Successive discrimination reversal (SDR) performances of Chukars, Ringneck doves and Greater Hill Mynas as a function of correction as opposed to non-correction procedures
}

\author{
ROBERT L. GOSSETTE ANO PATRICIA HOOD \\ COMPARATIVE ANIMAL BEHAVIOR LABORATORY, HOFSTRA UNIVERSITY
}

The performances of Chukars, Ringneck doves and Greater Hill mynas on successive discrimination reversals were studied under conditions of correction and non-correction. Correction procedures significantly elevated the error scores of the doves and mynas, but did not affect Chukar scores.

Over the past decade, there has been a growing interest in the use of successive discrimination reversal (SDR) in the comparative analysis of behavior. It now appears that SDR measures provide one of the more successful procedures for calibrating interspecies differences. Unfortunately, until recently direct inter-species comparisons, with identical task and methodology (Gossette, Gossette, \& Riddell, 1966; Gossette, 1966) have rarely been attempted. The bulk of the earlier studies limited their conclusions to a single species and to a statement of whether or not it displayed a reduction of errors across reversal problems. While most of these studies differed with respect to many methodological variables, one procedural variable of considerable interest is the use of correction as opposed to noncorrection procedures.

Both procedures have been used across widely separated vertebrate and invertebrate species, but there has been no systematic study of the effect of this procedural variable upon SDR measures. However, it would appear that correction should accelerate reduction of errors across successive reversals, especially during the earlier problems when errors predominate, since each error is necessarily followed by a correction or reinforceable response. Further, the effect of correction should be amplified with some organisms, such as the "lower" species, producing greater error scores.

Method

To test this hypothesis, three species of birds, representing a high, an intermediate, and a low SDR reduction level in a spatial SDR task were tested under conditions of correction and noncorrection.

Groups of six Chukar, eight ringneck doves, and eight Greater Hill mynas were divided to form a correction and noncorrection condition. All Ss were between one and two years of age and had been maintained in the laboratory for at least six months prior to testing.

All training was given in a formboard box, $28 \mathrm{in}$. long, $12 \mathrm{in.} \mathrm{wide,} \mathrm{and} 14 \mathrm{in.} \mathrm{high.} \mathrm{An} \mathrm{enclosed} \mathrm{stim-}$ ulus block platform, containing two round foodwells, was located at one end of the box. Access to each foodwell by $S$ from within the test chamber was possible through a port directly in front of each foodwell. A gullotine door separated the foodwells from $S$. The interior of the box was lined with acoustical tile. Two white, round blocks, $1-1 / 2$ in. in diameter and $1 / 8$ in. thick served as stimuli.

All Ss were placed on a $23 \mathrm{~h}$ deprivation schedule and given habituation to the test chamber for about 30 days. While in the test chamber, Ss were permitted to eat from both foodwells. As Sbecame adapted to the chamber and ate readily from the foodwells, the two stimulus blocks were introduced; both foodwells were successively covered until block pecking to obtain food was strongly established. Preference testing and forced responding to the nonpreferredblock position were next conducted in order to equalize responding. Daily sessions of 20 trials were given to each $\mathrm{S}$.

Training of the original position habit was then initiated. Food was left under the nonpreferred block position. For the noncorrection groups, the guillotine door was not lowered until $S$ had dislodged the block covering the food. Training was continued until a criterion of two or fewer errors within a given 20 trial session had been achieved. At that point the reinforcement contingencies were reversed, and reversal training was initiated. Reinforcement consisted of grains of chicken feed (Chukars), pigeon feed (pigeons), and a small piece of grape (myna).

\section{Results and Discussion}

Originally, it had been planned to continue reversal testing until a minimum of 19 reversals had been completed for each species group. However, as the general trend of the data became obvious, testing was terminated. Thus, the doves and mynas completed 14 problems, while the Chukars completed only 10 problems. The principal measure selected for analysis was the errors per problem across the first 10 problems. Analysis of variance by a 2 by 3 factorial design revealed significant differences among species $(p<.05)$, and between procedures $(p<.01)$. As can be readily seen from Fig. 1 , the dove and myna correction groups produced far more errors than their noncorrection controls, with little difference between the two Chukar groups. In addition, within 


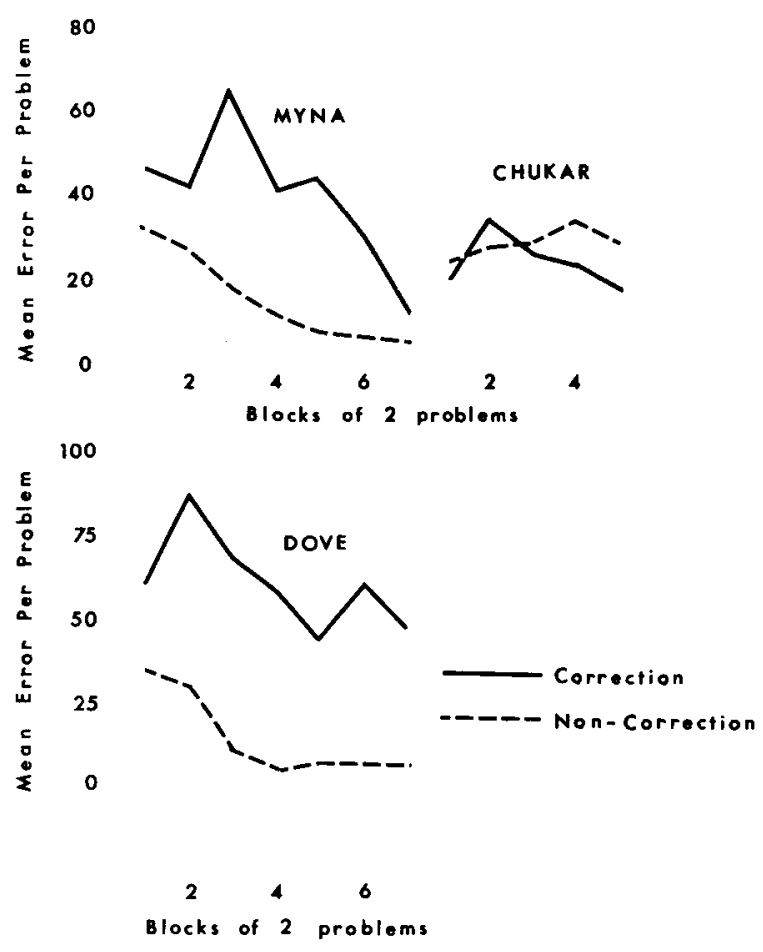

Fig. 1. Mean error for three avian species under conditions of correction and noncorrection.

each procedural condition the mynas produced fewer errors than the doves.

Observation of the behavior of mynas and doves during testing immediately suggested one hypothesis to account for the heightened errors for the correction groups. It seemed obvious that following several sessions on a given problem, a strong response chain became established between pecking the incorrect block and the correct block. While no objective, quantitative data are available for analysis, it was quite apparent that the amplitude of the incorrect response progressively declined until $S$ pecked with insufficient force to move the stimulus block, and thus failed to produce an error. But such abortive error behavior generally persisted throughout testing. The Chukars failed to display much of this "chaining." Interestingly, the time separating an incorrect response and the subsequent correction response was typically quite short (e.g., 1-3 sec) for the doves and mynas, but long (e.g., 5-10 sec) for the Chukar.

A serious question is posed by the effect of correction upon SDR error functions. Our original hypothesis argued for increased rate of error reduction when correction was employed. Setterington \& Bishop (1967) have recently reported a SDR study with African mouthbreeder fish in which unlimited correction, as opposed to "guidance" or restricted correction (as used by Behrend et al (1965) and others) was provided. Most significantly, opportunity for correction was provided 2 sec after each incorrect response. Unlike all other previous SDR studies with fish, these authors did find significant error reduction across reversals. Thus their findings would appear to support our basic hypothesis. It appears that the $2 \mathrm{sec}$ separation of an error and its subsequent correction, which we will call the correction interval, prevented the emergence of chaining that is believed to have elevated the error functions of the myna and dove groups in the present experiment. Detailed parametric studies of the correction interval are needed to permit evaluation of the power of correction in yielding more rapid discrimination learning. Such information is especially crucial since some writers (Bitterman, 1965) have proposed that the failure of fish to display error reduction under conditions of noncorrection or even "guidance" demonstrates a qualitative rather than a quantitative difference between the learning capacities of fish (and possibly turtles) and higher vertebrates.

The basic rationale underlying the use of correction has been that during the early phases of reversal, the excessive nonreinforcement occasioned by persistent errors would extinguish all responding, especially among the "lower"' species. To date we have studied SDR performances of 10 avian and six mammalian species, and have found such a tendency with only one species, Black-neck stilts. However, a brief reshaping quickly restored responding. The fact that other investigators have reported noncorrection SDR performances with even lower species, including invertebrates, argues against the necessity of correction in SDR studies.

\section{References}

Behrend, Erika, Domesick, Valerie \& Bitterman, M. E. Habit reversal in the fish. J. comp. physiol. Psychol., 1965, 60, 407-411.

Bitterman, M. E. The evolution of intelligence. Scient. American, $1965,212,92-100$.

Gossette, R., Gossette, Madeliene, \& Riddell, W. Comparisons of successive discrimination reversal performances among closely and remotely related avian species. Anim. Behav., 1966, 14, 560564.

Gossette, R. Comparison of successive discrimination reversal performances across 14 different avian and mammalian species. Amer. Zool., 1966, 6, (Abstract).

Setterington, R. G., \& Bishop, H. E. Habit reversal improvement in the fish. Psychon. Sci., 1967, 7, 41-42.

\section{Notes}

1. We wish to thank Miss Gail Krause for assistance in gathering data reported in this experiment.

2. This work was supported by NIMH grant MN 111806-01. 